\title{
The four dimensions: a model for the social aetiology of psychosis
}

Jai Shah, Romina Mizrahi and Kwame McKenzie

\section{Summary}

Recently, there has been increasing focus on prevention of mental illness, early intervention and the promotion of mental health. The social determinants of health and public health approaches are considered key. Early intervention has focused on psychotic disorders but prevention has not. This may in part reflect the fact that public health planners do not have a clear model for how social determinants influence the risk of developing a psychotic illness. Drawing on biological, genetic and epidemiologic evidence regarding the relationship between social risk factors and psychosis, this paper outlines a conceptual framework for understanding how individual and ecological factors contribute and interact to modulate the risk of developing psychotic illness. The framework asserts that there are four dimensions: individual factors; ecological factors; the interaction between individual and ecological factors; and time. It may help those considering interventions to understand the multilevel and multifactorial effects of social factors on the aetiology of psychotic illness, to develop targeted strategies for the prevention of psychotic illness and serve as a template for the assessment of initiatives.

\section{Declaration of interest}

None.
The World Health Organization (WHO) has concluded that prevention of mental illness is a public health priority. ${ }^{1}$ Effective prevention can reduce the risk of mental disorders, but to date the most severe forms of mental illness have not been the target of major prevention efforts. Schizophrenia and other psychoses are substantial public health problems that have a profound impact on national economies, health and social systems, affected individuals and their families and carers. Specific prevention initiatives remain elusive. This may in part be because models of prevention tend towards a population health approach and focus on the impact of social determinants on health. Although it is generally accepted that social risk factors are important in the genesis and maintenance of common mental disorders and affective states, there has been less acknowledgment of their role in the aetiology of psychotic states. There is now, however, a growing literature outlining the associations between a number of social or societal variables and the onset, course and outcome of psychosis. And yet - significantly for policymakers and public health planners - there is thus far no agreed model or framework regarding how various social factors may modulate the risk of psychotic illnesses that could aid in the development and targeting of public health interventions and further research.

The social world is hard to investigate. Factors can have effects at a single level or multiple social levels - individual, family, group, city or country. The effects can be proximal to the exposure or there may be a significant delay, a latent effect, which makes measurement difficult. Social factors may trigger a chain of events that increase risk over a life course. And different factors can interact - decreasing or amplifying each others' effects. A further complication is offered by March et al, ${ }^{2}$ who make the distinction between conditions (such as being unemployed) and social processes (such as the economic factors that lead to unemployment and make the experience of being unemployed unpleasant). Both may be related to the development of mental health problems but different interventions may be required to change their effects. Any interventions should be built on the growing understanding of how social factors may increase the risk of developing a psychotic illness. ${ }^{3,4}$

\section{Social factors and psychotic illness}

There are a variety of explanatory models for the impact of social factors on psychotic illness. Social factors may change the exposure to or action of biological risk factors or they may have a direct toxic effect on individuals and their families. Genetic and epigenetic processes interacting with social factors at the individual, group and societal levels over a life course produce a complex and interconnected web of causation. ${ }^{3,4}$

Understanding how different social factors change the risk of psychosis has been problematic, in part because of the numerous possible mechanisms and multiple levels through which this could occur. $^{3-5}$ For instance, there is a reported association between prenatal malnutrition and an increased risk of schizophrenia in adolescent life. This could reflect the impact of nutritional stress on the hypothalamic-pituitary-adrenal (HPA) axis or direct effects of the low levels of nutrients on deoxyribonucleic acid stability or expression (to name just two theories). However, at a different level, urban or rural residence during famine may change the risk of exposure by altering access to food to supplement the diet. ${ }^{5}$

The complex nature of this enquiry is demonstrated here by consideration of one of the explanatory models for the impact of social factors on psychotic illness: the 'vulnerability-stress model.', ${ }^{6,7}$ This model posits that genetic or developmental vulnerabilities interact with social adversity to culminate in a common pathway leading to stress. ${ }^{8,9}$

Genetic, biochemical and neurological evidence supports the link between stress and psychosis. ${ }^{10-12}$ The physiological effects of stress are demonstrated by findings of activation of the HPA axis to produce excess cortisol and adrenocorticotropic hormone. ${ }^{13}$ In addition to genetic factors, acquired vulnerabilities such as prenatal exposures, perinatal complications, drug misuse and childhood abuse or neglect may regulate the response to stress. Links between the physiology of stress and that of psychosis have been reported. The hippocampus, known to play a key role in dampening HPA activity, is reduced in volume in psychotic disorders. ${ }^{14-17}$ Stress also leads to mesolimbic release 
of, dysregulated mesocortical response to and overall sensitisation to dopamine, a neurotransmitter that may mediate psychotic symptoms. ${ }^{18-20}$

Experimental support for this is found, for example, in studies in which psychotic symptoms are associated with increased dopamine release after amphetamine challenge. ${ }^{21}$ Over time, in individuals who are at risk, exposure to moderate levels of stress can lead to an excessive and persistent dopamine response. Building on this theory, those with higher rates of life stressors may be at particular risk of developing a psychotic illness.

Epidemiological studies support this at multiple levels. Cumulative exposure to traumatic life events, or the number of life events experienced, is associated with an increased risk for psychosis $^{22-25}$ and the British National Psychiatric Morbidity Survey has reported that adverse life events are associated with subsequent psychotic experiences in the general population. ${ }^{26,27}$ In addition to traumatic major life events, the accumulation of minor events or 'daily hassles' have also been linked to psychotic illness. $^{28}$

However, it is not just the accumulation of life events that is important. As stated previously, a life event may trigger a train of other events. For instance, loss of a parent as a child (which has been associated with increased risk of later psychosis) could increase the chance of living in poverty and thus exposure to other environmental risk factors associated with psychosis.

At the level of gene-environment interaction, minor life events and daily hassles interact with polymorphisms known to be involved in dopamine neurotransmission to cause differential stress reactivity and psychotic experiences. ${ }^{29}$ Of note, the same polymorphism has been shown to correlate with differential risk for psychosis in individuals who use cannabis, ${ }^{30}$ further highlighting the complex interplay between multiple biological and environmental risk factors. And genetic variants and environmental exposures can interact in ways that are protective and not just harmful. Instead of focusing on 'vulnerability genes' that confer increased risk in the presence of certain environments, Belsky et al call for an appreciation of 'plasticity genes' that confer a nuanced differential susceptibility: increased risk in some environments, and decreased risk in others. ${ }^{31}$

Physiologically and cognitively, exposure to early developmental stressors (such as childhood trauma) may act by sensitising people to later adverse events, major or minor. They may increase the likelihood of adverse events for instance by shaping the capacity of individuals to form relationships. ${ }^{7,32}$ Alternately, they may interface with a person's attributional style - potentially making them more prone to psychotic thinking. ${ }^{33}$

Moving from the individual to the ecological, a major branch of investigation into the social aetiology of psychotic illness began in 1939 with Faris \& Dunham's work on the urban environment. ${ }^{34}$ Since then, research has focused on better defining its role as a risk factor for psychosis. Studies have demonstrated that the higher risk of psychotic illness for those living in cities is not the result of social drift but is associated with being born and growing up in an urban environment in childhood. Moreover, the bigger the city the bigger the risk. ${ }^{35-38}$

Living in an urban environment must, however, be a marker for a number of exposures. Allardyce et al found that after adjusting for social fragmentation and deprivation, there was only a non-significant trend towards an association between urbanicity and schizophrenia. ${ }^{36}$

Other factors may be important for the association between place and psychosis. For instance, Silver et al documented that the proportion of people moving in and out of an area was associated with higher rates of schizophrenia. ${ }^{39}$ Another pilot study has reported that increased social cohesion and social efficacy in areas in London, UK were associated with a reduced incidence of psychosis, ${ }^{40}$ and after controlling for individual deprivation, Boydell et al have shown an increased rate of schizophrenia with increasing neighbourhood inequality, but only in more deprived areas. ${ }^{41}$

Kirkbride et al recently used multilevel Poisson regression simultaneously to model individual- and neighbourhood-level factors. $^{42}$ They reported that $23 \%$ of the incidence of schizophrenia could be attributed to neighbourhood-level social risk factors including socioeconomic deprivation, voter turnout (proxy for social capital), ethnic fragmentation (proxy for segregation) and ethnic density (95\% CI 9.9-42.2). A 1\% increase in voter turnout (incidence rate ratio (IRR) $=0.95,95 \%$ CI 0.92 0.99 ) and ethnic segregation (IRR $=0.95,95 \%$ CI $0.92-0.99$ ) were both independently associated with a reduced incidence of $5 \%$, independent of age, gender, ethnicity, deprivation and population density. ${ }^{42}$

However, there are a number of different mechanisms through which ecological factors could have their impact on psychosis. Stress may be just one of a number of mechanisms. ${ }^{5}$

Taken as a whole, the literature demonstrates that social factors are associated with psychosis risk at an individual and an ecological level. There is evidence of an increased risk with greater exposure to some individual risk factors and with a greater number of risk factors. There are multiple mechanisms and an interplay between genetic, epigenetic and environmental factors.

\section{Conceptual framework}

A framework for categorising or organising different associations between psychotic illnesses and risk factors may be helpful to those who are considering developing prevention/promotion initiatives. It may also further research in the field. Although we have highlighted the stress hypothesis, this umbrella conceptual framework we present would allow the inclusion of a variety of perspectives on causation and different mechanisms. A simplified conceptual model would follow what we know from the literature and posit that there are four dimensions of interest:

(1) exposure to individual-level social factors linked to psychosis;

(2) exposure to ecological-level social factors linked to psychosis;

(3) the interaction between individual and ecological social factors; and

(4) time.

\section{Exposure to individual-level social factors linked to psychosis}

At the individual level, the model would be similar to heart disease: there is an inherited risk, but whether one develops a heart attack or not depends on other risk, protective and health promotion factors that one encounters. Accordingly, the risk of developing a psychosis for any individual would depend in part on inherited vulnerabilities, but also would rest on the balance between exposure to factors that either increase or decrease risk or enhance mental health. In addition to genetic risk and biological risk factors, a list of social risk factors could include the use of certain drugs (especially cannabis), racial discrimination and factors in development such as bullying and psychological trauma in childhood, separation from parents and other childhood adversity. ${ }^{41}$ The amount of exposure or number of factors to which a person is exposed would need to be sufficient for a psychotic illness to develop. Previous work has shown that 
the greater the number of risk factors the higher the risk of psychosis. $^{22-27}$

\section{Exposure to ecological-level social factors linked to psychosis}

At the ecological level social factors change the risk on a population basis or change environmental risk. The model would be similar to that of diabetes, where a change in the availability and calorific content of certain types of food and cultural changes in exercise have led to increased rates of the illness. Groups with similar individual-level risk factors may have different rates of illness dependent on the environment. Environmental risk factors have been less well studied but they would include city birth and city living, famine, social cohesion, social fragmentation, minority groups living in areas with lowpopulation densities of their group, and migrants from countries that are predominantly Black living in countries that are predominantly White. ${ }^{32-41}$

\section{Interaction between individual and ecological social factors}

However, in the real world individual and ecological risks may interact. For instance, some ecological factors could decrease the rates of illness, either by decreasing the impact of individual risk factors or by reducing the possibility of exposure to individual social risks. The social safety net, for instance, may mitigate the impact of life events. Conversely, some ecological factors could interact with other ecological or individual factors to increase risk. Individual and ecological factors may not simply be independent variables acting in synergy, but may amplify each others' effects thus further increasing risk. For example, at an individual level use of cannabis may increase a person's risk of developing a psychotic illness. But at an ecological level, the availability of cannabis in the community could increase the risk of that individual using cannabis in the first place. The social context is also important in other ways. Regular use of cannabis in some jurisdictions may offer access to a subculture or environment which changes the risk of exposure to other social factors associated with psychosis. The environment of some regular drug users is characterised by more daily hassles and life events than that of the rest of the public. Exposure to more life events and daily hassles may lead to higher levels of perceived stress and thus further increasing the risk of psychosis. In this way, environment and individual-level risk factors may amplify each other.

\section{Time}

The fourth dimension is time and this can be considered in a number of different ways. First, sufficient exposure to an individual or ecological risk factor may only occur over time. Second, time may be needed for the interaction between individual and ecological risk factors to amplify. Third, there are sensitive periods in brain development at which time exposure to certain risk factors may be more important. For instance, being born and brought up in a city is aetiologically more significant in schizophrenia than living in a city per se and other risk factors such as separation from parents may be more important in childhood than adult life. Fourth, there may be a delay in time between the exposure to a risk factor and the development of a psychosis. For instance, the impact of maternal malnutrition on psychosis risk may only be evident when offspring reach early adult life. ${ }^{5}$

\section{Developing interventions}

This simplified model may be useful in considering possible prevention and promotion initiatives, since each dimension can be linked to types of strategies. Prevention at an individual level will aim to decrease the amount of exposure or limit the number of different social factors to which a person is exposed. Promotion efforts would enhance behaviours and expose individuals to social factors known to strengthen mental health. At an ecological level, the aim would be to develop healthy environments and limit exposure to psychologically noxious social environments. Where interactions between individual-ecological factors lead to an amplification or spiralling of risk, targeting such interactions may be important. If neither individual risk factors nor environmental risk factors can be reduced, then remedial strategies aimed at uncoupling the links between factors could be effective. And, of course, time is all-important. Understanding the stages of development at which particular risk factors act is important for targeting prevention strategies for maximal effect. Understanding the longer-term impacts of particular risk factors may give an indication of how long preventive strategies should last. Finally, understanding the timescales over which social factors may have their effects helps evaluators to pick the right time frame for assessment of the effectiveness of interventions.

\section{Conclusion}

We have outlined here a framework for considering how a range of social factors, acting over time, could individually or together make a contribution to the development of psychotic illness. The importance of such a model for conceptualising the social aetiology of psychosis lies in demonstrating the complex and multifaceted interplay between individual and ecological dimensions, their interactions, and time, while taking into account gaps in knowledge or the current evidence base. We hope that this model will help in research; in the design and planning of interventions aimed at particular risk and protective factors; in the training of health professionals; and in the development of health and social systems that more effectively support populations at risk of developing psychosis.

Jai Shah, MD, BSc, MSc, Department of Psychiatry, Harvard University, Cambridge, Massachusetts, USA; Romina Mizrahi, MD, PhD, PET Centre, Centre for Addiction and Mental Health, Toronto and Department of Psychiatry, University of Toronto: Kwame McKenzie, BM, MRCPsych, University of Toronto and Centre for Addictions and Mental Health, Toronto, Canada

Correspondence: Kwame McKenzie, Professor of Psychiatry, Centre for Addictions and Mental Health, Suite 300, 455 Spadina Avenue, University of Toronto, Toronto M5S 2G8, Canada. Email: kwame_mckenzie@camh.net

First received 10 Apr 2010, final revision 6 Dec 2010, accepted 23 Feb 2011

\section{References}

1 Hosman C, Jane-Llopis E, Saxena S. Prevention of Mental Disorders: Effective Interventions and Policy Options. Oxford University Press, 2005.

2 March D, Morgan C, Bresnahan M, Susser E. Conceptualising the social world. In Society and Psychosis (eds C Morgan, K McKenzie \& P Fearon): 41-57. Cambridge University Press, 2008.

3 Kirkbride J, Jones PB. The prevention of schizophrenia-what can we learn from eco-epidemiology? Schizophr Bull 2011; 37: 262-71.

4 March D, Susser E. Invited commentary: taking the search for causes of schizophrenia to a different level. Am J Epidemiol 2006; 163; 979-81.

5 Susser E, St Clair D, He L. Latent effects of prenatal malnutrition on adult health: the example of schizophrenia. Ann NY Acad Sci 2008; 1136: 185-92. 
6 Nuechterlein KH, Dawson ME. A heuristic vulnerability/stress model of schizophrenic episodes. Schizophr Bull 1984; 10: 300-12.

7 Myin-Germeys I, Van Os J. Adult adversity: do early environment and genotype create lasting vulnerabilities for adult social adversity in psychosis? In Society and Psychosis (eds C Morgan, K McKenzie \& P Fearon): 127-42. Cambridge University Press, 2008.

8 Miller $\mathrm{P}$, Lawrie SM, Hodges A, Clafferty R, Cosway R, Johnstone EC. Genetic liability, illicit drug use, life stress and psychotic symptoms: preliminary findings from the Edinburgh study of people at high risk for schizophrenia. Soc Psychiatry Psychiatr Epidemiol 2001; 36: 338-42.

9 Mason O, Startup M, Halpin S, Schall U, Conrad A, Carr V. Risk factors for transition to first episode psychosis among individuals with 'at-risk mental states'. Schizophr Res 2004; 71: 227-37.

10 Walker EF, Diforio D. Schizophrenia: a neural diathesis-stress model. Psycho Rev 1997; 104: 667-85.

11 Walker E, Mittal V, Tessner K. Stress and the hypothalamic pituitary adrena axis in the developmental course of schizophrenia. Annu Rev Clin Psychol 2008; 4: 189-216.

12 Jones SR, Fernyhough C. A new look at the neural diathesis-stress model of schizophrenia: the primacy of social-evaluative and uncontrollable situations. Schizophr Bull 2007; 33: 1171-7.

13 Ryan MC, Sharifi N, Condren R, Thakore JH. Evidence of basal pituitaryadrenal overactivity in first episode, drug naive patients with schizophrenia. Psychoneuroendocrinology 2004; 29: 1065-70.

14 Steen RG, Mull C, McClure R, Hamer RM, Lieberman JA. Brain volume in firstepisode schizophrenia: systematic review and meta-analysis of magnetic resonance imaging studies. Br J Psychiatry 2006; 188: 510-8.

15 Geuze E, Vermetten E, Bremner JD. MR-based in vivo hippocampal volumetrics: 2. Findings in neuropsychiatric disorders. Mol Psychiatry 2005; 10: $160-84$.

16 Lieberman J, Chakos M, Wu H, Alvir J, Hoffman E, Robinson D, et al. Longitudinal study of brain morphology in first episode schizophrenia. Biol Psychiatry 2001; 49: 487-99.

17 Fusar-Poli P, Perez J, Broome M, Borgwardt S, Placentino A, Caverzasi E, et al. Neurofunctional correlates of vulnerability to psychosis: a systematic review and meta-analysis. Neurosci Biobehav Rev 2007; 31: 465-84.

18 Soares JC, Innis RB. Neurochemical brain imaging investigations of schizophrenia. Biol Psychiatry 1999; 46: 600-15.

19 Seeman P, Kapur S. Schizophrenia: more dopamine, more D2 receptors. Proc Natl Acad Sci USA 2000; 97: 7673-5.

20 Kapur S. Psychosis as a state of aberrant salience: a framework linking biology, phenomenology, and pharmacology in schizophrenia. Am J Psychiatry 2003; 160: 13-23.

21 Laruelle M, Abi-Dargham A. Dopamine as the wind of the psychotic fire: new evidence from brain imaging studies. J Psychopharmacol 1999; 13: 358-71.

22 Shevlin M, Houston JE, Dorahy MJ, Adamson G. Cumulative traumas and psychosis: an analysis of the national comorbidity survey and the British Psychiatric Morbidity Survey. Schizophr Bull 2008; 34: 193-9.

23 Selten JP, Cantor-Graae, E. Social defeat: risk factor for schizophrenia? Br J Psychiatry 2005; 187: 101-2.

24 Selten JP, Cantor-Graae E. Hypothesis: social defeat is a risk factor for schizophrenia? Br J Psychiatry 2007; 191 (suppl 51): s9-12.

25 Cantor-Graae E. The contribution of social factors to the development of schizophrenia: a review of recent findings. Can J Psychiatry 2007; 52: 277-86.
26 Johns, LC, Cannon M, Singleton N, Murray RN, Farrell M, et al. Prevalence and correlates of self-reported psychotic symptoms in the British population. Br J Psychiatry 2004; 185: 298-305.

27 Wiles NJ, Zammit S, Bebbington P, Singleton N, Meltzer H, Lewis G. Self-reported psychotic symptoms in the general population: results from the longitudinal study of the British National Psychiatric Morbidity Survey. Br J Psychiatry 2006; 188: 519-26.

28 Myin-Germeys I, van Os J. Stress-reactivity in psychosis: evidence for an affective pathway to psychosis. Clin Psychol Rev 2007; 27: 409-24.

29 van Winkel R, Henquet C, Rosa A, Papiol S, Fananás L, De Hert M, et al Evidence that the COMT(Val158Met) polymorphism moderates sensitivity to stress in psychosis: an experience-sampling study. Am J Med Genet $B$ Neuropsychiatr Genet 2008; 147B: 10-7.

30 Caspi A, Moffitt TE, Cannon M, McClay J, Murray R, Harrington H, et al. Moderation of the effect of adolescent-onset cannabis use on adult psychosis by a functional polymorphism in the catechol-O-methyltransferase gene: Iongitudinal evidence of a gene $\times$ environment interaction. Biol Psychiatry 2005; 57: 1117-27.

31 Belsky J, Jonassaint $C$, Pluess $M$, Stanton M, Brummett B, Williams R. Vulnerability genes or plasticity genes? Mol Psychiatry 2009; 14: 746-54.

32 Wright J, Turkington D, Kingdon DG, Ramirez Basco M. Cognitive-Behavior Therapy for Severe Mental Illness: An Illustrated Guide. American Psychiatric Publishing, 2009.

33 Bentall RP, Kinderman P, Kaney S. The self, attributional processes and abnormal beliefs: towards a model of persecutory delusions. Behav Res Ther 1994; 32: 331-41.

34 Faris R, Dunham H. Mental Disorders in Urban Areas. University of Chicago Press, 1939.

35 Spauwen J, Van Os J. The psychosis proneness: psychosis persistence model as an explanation for the association between urbanicity and psychosis. Epidemiol Psichiatr Soc 2006; 15: 252-7.

36 Allardyce J, Gilmore H, Atkinson J, Rapson T, Bishop J, Mccreadie RG. Social fragmentation, deprivation and urbanicity: relation to first-admission rates for psychoses. Br J Psychiatry 2005; 187: 401-6.

37 van Os J, Hanssen M, Bijl RV, Vollebergh W. Prevalence of psychotic disorder and community level of psychotic symptoms: an urban-rural comparison. Arch Gen Psychiatry 2001; 58: 663-8.

38 Boydell J, McKenzie K. Society, place and space. In Society and Psychosis (eds C Morgan, K McKenzie \& P Fearon): 77-94. Cambridge University Press, 2008.

39 Silver E, Mulvey E, Swanson J. Neighbourhood structural characteristics and mental disorder: Faris and Dunham revisited. Soc Sci Med 2002; 55 1457-70.

40 Boydell J, van Os J, McKenzie K, Allardyce J, Goel R, Mccreadie RG. Incidence of schizophrenia in ethnic minorities in London: ecological study into interactions with environment. BMJ 2001; 323: 1336-8.

41 Boydell J, van Os J, McKenzie K, Murray RM. The association of inequality with the incidence of schizophrenia - an ecological study. Soc Psychiatry Psychiatr Epidemiol 2004; 39: 597-9.

42 Kirkbride JB, Morgan C, Fearon P, Dazzan P, Murray RM, Jones PB. Neighbourhood-level effects on psychoses: re-examining the role of context. Psychol Med 2007; 37: 1413-25. 